\title{
Essay:
}

\section{Geborgte Narrative: Wie sich türkische Einwanderer an den Juden in Deutschland orientieren.}

\section{Von Y. Michal Bodemann und Gökce Yurdakul}

Von ihren Anfängen, vor allem von der Chicagoer Schule her, hat die nordamerikanische Soziologie im Bereich der Ethnizitäts- und Migrationsforschung untersucht, wie sich Einwanderergruppen integrieren: in bestimmten städtischen Räumen, Regionen und Staaten, in wirtschaftlichen, kulturellen und Klassenstrukturen, und wie sich Einwanderer im Laufe der Generationen assimilieren. Selten jedoch wurde dabei überlegt, wie ethnische Gruppen ihre Integration durch ihre Führung und durch ihre Organisationen selbst mitbestimmen, und zwar nicht nur gegenüber Staat und Zivilgesellschaft allgemein, sondern insbesondere auch im Bezug auf andere Immigrantengruppen oder Minoritäten. Einwanderer freilich treten nicht nur spontan miteinander in Beziehung und orientieren ihr Verhalten wechselseitig aneinander, sondern oft sind es die jüngeren Einwanderergruppen, die die Narrative der älteren Gruppen zum Modell nehmen; andererseits sind es gelegentlich auch ältere Einwanderergruppen, die sich in ihren Forderungen, einschließlich nach vorzugsweiser Behandlung, auf jüngere Einwanderergruppen beziehen. Eines der bekanntesten Beispiele dafür, wie Mitglieder einer Gruppe das Narrativ einer anderen Gruppe übernehmen und sich damit identifiziert haben, ist das Engagement amerikanischer Juden mit der NAACP und der Bürgerrechtsbewegung in den USA ${ }^{1}$.

Im folgenden fragen wir aus diesem Blickpunkt, wie artikulieren sich interethnische Beziehungen zwischen Türken ${ }^{2}$ und Juden in Deutschland? Wie konnte sich, vor allem mit bezug auf die Türken, aus dem Nichts heraus eine Einwanderergruppe organisieren und strukturieren? Welche Ziele stehen ihr vor Augen und welche Modelle und Narrative haben sich bewährt? Zweifellos steht für ihre Führung, gleich welcher Orientierung, ob religiös oder säkular, ein spurenloses Verschmelzen mit der deutschen Bevölkerung außer Frage, denn sie wollen sich als eigenständige Ethnie behaupten. Organisatorische Muster aus dem Herkunftsland erweisen sich schnell als unbrauchbar: Die diasporische Situation in Deutschland, das Verhältnis zu Staat und Politik sind gänzlich anders. Doch im Gegensatz zu den meisten klassischen Einwandererländern gibt es hierzu in Deutschland nur wenige Vorbilder - oder genauer: es gibt nur eines, nämlich das der jüdischen Gemeinschaft. Die Juden wiederum hatten zwar über die Jahrhunderte verschiedene Varianten ihres diasporischen Narrativs in Deutschland entwickelt, doch mussten auch sie sich nach der Schoah in West- und Ostdeutschland neu organisieren und drastisch veränderte Narrative entwickeln. Als Modell freilich standen jüdische Organisationsstrukturen und diese Narrative den türkischen Neuankömmlingen sodann bereits zur Verfügung ${ }^{3}$.

1) Freilich war das jüdische Engagement in der Bürgerrechtsbewegung von eher ungewöhnlicher, »altruistischer « Form und definierte sich als Unterstützung der Schwarzen. Doch gleichzeitig war dieses Engagement auch für die gesellschaftliche Position der jüdischen Minorität nicht unwichtig, angesichts persistierender Diskriminierungen gegen Juden bis tief in die sechziger Jahre. Siehe hierzu auch Ezra Mendelsohn (1993, S.133ff).

2) Wenn nicht anderweitig bezeichnet, verstehen wir unter »Türken« hier diejenigen Menschen, die ihre Herkunft aus der Türkei herleiten und sich als »Türken« betrachten. Dies mag Menschen kurdischer, arabischer, aramäischer usw. Herkunft ausschließen.

3) Zur Entwicklung des neuen deutschen Judentums und jüdischer Narrative nach 1945 steht eine reichhaltige Literatur zur Verfügung. Siehe hier zum Einstieg vor allem Michal Bodemann (1996, 2002, 2005), Michael Brenner (1995), Dan Diner (1986), Burgauer (1993) und für die DDR, Ulrike Offenberg (1998).

Soziale Welt 56 (2005), S. 441 - 452 
Der Begriff Diaspora selbst beschreibt ursprünglich die Zerstreuung der Juden nach dem babylonischen Exil, Zerstreuung auch in dem positiven Sinne von Aussaat, und wurde noch in der Antike als Vorbild auch auf Griechen und Armenier angewandt. Aus heutiger Perspektive ist die Diaspora eine in Migration und Transnationalität aufgehobene Gemeinschaft, die sich mit statischen Begriffen kaum identifizieren lässt. Tatsächlich hat sich die türkische Einwanderergruppe auch mit ihren vielfältigen inneren ethnischen wie auch religiösen Brüchen, kurdischen, alewitischen, anatolischen und europäischen Ursprüngen auf ihrem Weg zur ethnischen Minorität in Deutschland bereits seit längerem dieses jüdischen Vorbilds bedient. Wir meinen, dass der Vorwurf, Türken bildeten Parallelgesellschaften, gerade mit dieser Entwicklung zur Diaspora hin zu tun hat ${ }^{4}$. Das Bild einer düsteren Hinterhofkultur mit Zwangsehen, Ehrentötungen und Hasspredigten ist dabei reichlich einseitig. Die Mehrheit der Türken hat sich in Deutschland pragmatisch-apolitisch eingerichtet und hat ihr traditionelles Verhalten gerade im Bereich der Geschlechterrollen nivelliert. Dementsprechend ist auch in der Führung unter Einwanderern ein Gottesstaat-Prediger im Stile Kaplans keineswegs typisch: typisch sind vielmehr jene, von denen weniger gesprochen wird: jüngere, in Deutschland aufgewachsene Geschäftsleute, Ärzte oder Rechtsanwälte, die längst dabei sind, ihrer Einwanderergruppe ein Deutschland angepasstes, modernes Gepräge zu geben und die gleichzeitig als Mittler zwischen ihrer Klientel und deutschen Stellen in Rechts- und Fürsorgefragen zur Verfügung stehen. Die Entwicklung dieser Einwanderergruppe wurde dabei in der Folgezeit wesentlich geprägt durch die Wende 1989 und den 11. September 2001.

\section{Die deutschen Türken: Zwischen Wende und 11. September}

Siebzehn Jahre nach der »Endlösung der Judenfrage « kamen die ersten türkischen Migranten nach Deutschland und nach Schätzungen des Statischen Bundesamtes leben knapp zwei Millionen türkische Staatsbürger aus insgesamt 7,3 Millionen Einwanderern im Land; 570000 Türken haben die deutsche Staatsbürgerschaft angenommen, also etwa ein Viertel der türkischen Einwanderung insgesamt. (Statistisches Bundesamt, TBB, 2000) Während sich, zwar zögerlich, mit dem neuen Staatsangehörigkeitsgesetz die rechtliche Lage der Einwanderer etwas gebessert hat, lässt sich dies über ihre wirtschaftliche Situation nicht behaupten. Mit dem Fall der Mauer und somit der Öffnung eines Billiglohnmarktes hat sich die Beschäftigungslage insbesondere der türkischen Bevölkerung, insbesondere auch in Berlin, drastisch verschlechtert. Viele Türken, die seit den 60er Jahren nach Deutschland gekommen waren, sind zunehmend auf Sozialhilfe angewiesen. Bereits Ende der 90er Jahre waren knapp 20\% der Ausländer in Berlin ohne Arbeit; somit sind sie doppelt so häufig arbeitslos wie die Deutschen (EFMS, 2000).

Das Problem der Arbeitslosigkeit wird verschlimmert durch die Diskriminierungen gegen Einwandererkinder im Bildungssystem. Deutsche Staatsbürger türkischer Herkunft beklagen sich, dass ihre Kinder im Bildungssystem benachteiligt werden (am Orde 2002). Während 8\% aller jungen Deutschen keine Ausbildungsplätze erhalten, sind es unter jungen Menschen türkischer Herkunft mit 40\% fünf mal so viele (Ausländerbeauftragte der Bundesregierung, 2000).

Wie andernorts in der westlichen Welt, so fiel von den kollabierenden World Trade Türmen ein dunkler Schatten auch auf alle Muslime in Deutschland. Überraschenderweise inten-

4) Notorisch unter diesen war die Titelgeschichte des Spiegel in der Ausgabe vom 15. November 2004, unter dem Titel »Allahs rechtlose Töchter. Muslimische Frauen in Deutschland « Andere Artikel hierzu waren u.a. von Navid Kermani in Die Zeit, 18. November 2004; Dick Pels, 23. November, Die Tageszeitung, Pascal Beucker, 22. November 2004, Die Tageszeitung, Jens Jessen, Die Zeit, 18. November 2004, Ulrich Beck und Michal Bodemann, beide in der Süddeutschen Zeitung vom 20./21. November 2004; Zafer Senocak, 22. November 2004, Die Tageszeitung; Hauptartikel der Berliner Zeitung, 15. November 2004; Annette Ramelsberger, 17. November 2004, Süddeutsche Zeitung; und über den Berlin-Neuköllner Bürgermeister Heinz Buschkowsky im Tagesspiegel, 13. November 2004. 
sivierte sich jedoch gleichzeitig der Antisemitismus. Viele Muslime, einschließlich türkische, beschuldigten die Juden bekanntlich als Drahtzieher: sie kolportierten die absurde, doch verbreitete Theorie, dass in den World Trade Towers arbeitende Juden über den Angriff Tage zuvor gewarnt worden seien und deshalb an diesem Tag nicht zur Arbeit aufgetaucht wären.

Andererseits jedoch sind die deutschen Türken, nicht weniger als in der Türkei selbst, alles andere als eine homogene Gruppe. Viele westlich orientierte Türken sind Juden gegenüber traditionell positiv eingestellt, während sich muslimisch orientierte Türken im israelischarabischen Konflikt eher auf die Seite der Palästinenser stellen. Dies wiederum schließt nicht aus, dass in der Türkei historisch starke Aversionen gegenüber der arabischen Welt existieren. Auch in Deutschland haben sich viele Türken mit den Juden zumindest unausgesprochen solidarisiert, um sich von den arabischen Einwanderern zu distanzieren ${ }^{5}$.

Trotz des Islams als Bindeglied sah sich die Türkei aufgrund der kemalistischen Modernisierung als Teil des Westens, und so gibt es auffallende Parallelen zwischen Israel und der (post-osmanischen) Türkei, die sich auch unter deutschen Türken artikuliert. Überdies verhielt sich die Politik verschiedener türkischer Regierungen der letzten Jahrzehnte vorsichtig pro-israelisch und tendenziell anti-arabisch. Die Türkei und Israel haben häufig kooperiert, einschließlich im Fall den Kurdenführers Öcalan, der 1999 mithilfe israelischer Agenten gefasst worden war (Turkish Daily News, 18 Februar 1999, Rubin 2001). Die pro-israelische Politik ist nicht denkbar ohne eine relative Offenheit oder zumindest Indifferenz in der türkischen Bevölkerung gegenüber Israel; umgekehrt beeinflusst die große Politik Stimmungen in der Bevölkerung. Die politischen Strömungen der Türkei wiederum finden ihren Nachhall auch unter deutschen Türken.

Als sich anti-arabische Sentimente in der Türkei durch die Synagogen-Anschläge in Istanbul im Jahre 2003 verschärften (Hürriyet, 15. November 2003), organisierte eine »Migrantische Initiative gegen Antisemitismus « zum 11. Jahrestag von Mölln in Berlin eine Demonstration. Diese betonte, die Juden stünden nicht allein im Kampf gegen Antisemitismus, und sie drückten ihre Solidarität mit Juden in Deutschland aus ${ }^{6}$. (Migrantische Initiative, 2003)

5) Die Freundschaft zwischen Israel und der Türkei hat eine lange Geschichte und kann als eine militärische Freundschaft und eine strategische Partnerschaft zusammengefasst werden. (Bir und Sherman 2002). Die freundschaftlichen Beziehungen des türkischen Staates zu Israel reflektieren nicht unbedingt die Ansichten der Türken selber. Antisemitismus ist in der Türkei weit verbreitet, insbesondere unter den religiösen und nationalistischen Gruppen (Milli Gazete, internet edition, 25. September 2005, siehe auch: Otuken.org, nihalatsiz.org). Wie auch immer, die anti-arabische Haltung ist auch nicht zu unterschätzen, besonders die pan-türkische Bewegung, die sich Anfang des 20. Jahrhunderts bildete (Hanioglu 2001). Zur Zeit haben türkische Nationalisten zwei deutliche Standpunkte gegen Araber: Zum einen argumentieren sie dafür, dass Araber-Sein und Muslim-Sein unterschieden werden soll (www.kurandakidin.net). Auch wenn Türken Muslime sind, distanzieren sie sich von den Arabern, indem sie die türkisch-islamische Synthese nachdrücklich betonen (Karesoglu 1999). Zum anderen argumentieren einige türkische Nationalisten, dass die authentische türkische Religion Schamanismus sei, und sie unterstreichen die Bedeutung zur Rückkehr der türkischen Wurzeln in Zentralasien. Daher sollen Türken nicht mit Islam oder Arabern assoziiert werden. Speziell nach dem 11. September wurden Türken Zielscheiben für anti-arabische Haltungen in Westeuropa und Nordamerika. Sie wollten sich von den Arabern distanzieren, um die Verleumdung zu vermeiden, die mit Muslim-Sein kommt. Die Worte Vural Ögers, eines prominenten deutsch-türkischen Geschäftsmannes und Mitglied des EU Parlaments, zeigen, wie sich die Türken von Arabern distanzieren wollen: „Die Akteure des politischen Islam sind keine Türken. Jihad ist keine türkische Sache. Palästina ist nicht das Problem der Türken, das wissen wir; einige Deutsche wissen das auch.» (Interview mit Vural Öger, 4. October 2005, Hürriyet/European Edition, Almanya).

6) Im November 2004 schließlich, quasi als Nachhall zu den Ereignissen des Vorjahrs, organisierte der TBB zusammen mit dem Jüdischen Kulturverein im Kreuzbergmuseum in Berlin eine »Fachtagung » unter dem Titel »Gemeinsam gegen Antisemitismus! Entwicklung von Handlungsstrategien für die Einwanderungsstadt Berlin «. 
Auch Safter Cinar vom Türkischen Bund Berlin-Brandenburg (TBB) schrieb später an das jüdische Gemeindeblatt, jüdisches berlin (j.b.) und drückte dort sein Mitgefühl mit den Juden Berlins aus (j.b. 2003).

In der nächsten Ausgabe des j.b. erschien sodann ein Artikel über Türken und türkische Juden, sowie Fotos einer gemeinsamen jüdisch-türkischen Hanukkah-Party, organisiert vom Jüdischen Kulturverein. Die Artikel bezogen sich auf das millet - System im Osmanischen Reich, wo Juden und andere Minoritäten in der türkisch-muslimischen Gesellschaft zusammen leben konnten (Tulgan 2004). Auch wurde hier betont, dass Juden sowohl nach der Spanischen Inquisition ab 1492 wie auch nach 1933 aus Deutschland im Osmanischen Reich und der Türkei Zuflucht gefunden haben (Shaw 1991). Hier nun bot das j.b. den Türken Raum, um ihre Solidarität mit Juden zu bezeugen (Yilmaz 2004). Der Berliner Jüdische Kulturverein tat sich in seinem Zusammengehen mit türkischen Gruppen besonders hervor.

Der 11. September stellte somit eine zweifache Herausforderung an die türkische Führung in Deutschland: sie musste versuchen, nicht unter den anti-arabisch/anti-muslimischen Hut $\mathrm{zu}$ geraten und musste gleichzeitig in ihrer eigenen Gefolgschaft die neue antisemitische Welle bekämpfen. Dieser neue Antisemitismus aber hatte zwei unterschiedliche Quellen - er fand sich nicht nur unter muslimischen Einwanderern, sondern auch unter den Rechten in Deutschland; unter den Rechten freilich verbunden mit zunehmendem Rassismus gegen Muslime.

Die Schmiererei an einer Wand in Berlin, die die Soziologin Riva Kastoryano notiert hatte, »Was die Juden hinter sich haben, haben die Türken noch vor sich« (Kastoryano 2002, S.132), wo wie so oft also Antisemitismus und Rassismus konvergieren, zwingt die deutschtürkische Führung praktisch in eine Allianz mit Juden, auch wenn diese erst zögerlich begonnen hat; sie folgt der Maxime, »die Feinde meiner Feinde sind meine Freunde«. Dementsprechend antwortet auch ein Foto, aufgenommen während eines Straßenprotestes in Berlin, und zeitweise im Jüdischen Museum Berlin ausgestellt, auf die Graffiti: In diesem Foto tragen türkische Einwanderer ein Plakat mit den Worten, »Wir wollen nicht die Juden von morgen sein. ${ }^{7}$ «Die Migranten antworten auf Neo-Nazis, indem sie das kulturelle Repertoire der deutsch-jüdischen Beziehungen positiv gebrauchen, für ihre ureigenen Zwecke ${ }^{8}$.

Hier nun wollen wir an einigen Fallbeispielen zeigen, wie sich türkisch-jüdische Beziehungen in Deutschland darstellen und wie die numerisch stärkste Einwanderergruppe, die Türken, die kleine jüdische Minorität mit ihrer herausragenden wie auch tragischen Rolle in der deutschen Geschichte als Modell ihrer eigenen Integration in die deutsche Gesellschaft nehmen. Riva Kastoryano (2002) hat diese Konstellation präzise formuliert: In Deutschland nehmen Türken die Juden als konkretes Beispiel einer Minorität, sowohl hinsichtlich der Geschichte wie auch der Organisation.

\section{Das Katzentor}

In der Ausgabe vom 23. September 2004 erschien im Wochenmagazin Der Stern eine Karikatur, von der zwar in der deutschen Öffentlichkeit kaum Notiz genommen wurde, die aber in der türkischen Gemeinschaft für große Empörung gesorgt hatte. Sie zeigte einen stereotypen älteren türkischen Mann mit finsterem Schnurrbart, der versuchte, durch das Katzenloch eines großen Tors zu kriechen. Das Tor war mit »Europäische Union« beschriftet und das

7) Das Transparent findet sich in Jonker (2002) abgebildet; die Tatsache, dass es in einem jüdischen Museum ausgestellt wird, zeigt die Affinität der beiden Minoritäten aufgrund ähnlich gelagerter Erfahrungen mit der deutschen Umwelt.

8) Zum Begriff des Repertoires, nach Charles Tilly, siehe Swidler (2002). 
Katzenloch mit einem Zweizeiler in imitiert-arabischer Schrift ornamentiert - obgleich die arabische Schrift im Türkischen bekanntlich nicht benutzt wird. Nicht nur eine orientalistische, sondern auch eine gängige anti-jüdische Grundstruktur ließ sich in der Karikatur erkennen: das Bild des jüdischen Untermenschen, der die deutsche Gesellschaft unterwandert. Vural Öger, prominenter deutsch-türkischer Geschäftsmann und Abgeordneter im EuropaParlament schrieb hierzu einen offenen Brief an den Stern und bezeichnete die Karikatur als diffamierend, obszön und als Futter für neo-nazistische Propaganda. Öger schloss seinen Brief wie folgt:

Ein junger Türke mit deutschem Pass, nicht allein hierzulande schon auf die Welt gebracht, nein, auch erzogen, hat im Geschichtsunterricht von Hitlers Anfängen gehört und dann gemeint, das sei eine Zeichnung, wie sie damals auch im "Stürmer" stand. Nur hätten die Juden andere Nasen bekommen. Hier im "stern" sei die Nase durch den Schnurrbart ersetzt worden, sonst aber sei das alles derselbe rassistische Mist. (Hürriyet, 2. Oktober 2004)

\section{Das Möllner »Novemberpogrom«}

Ein zweites Beispiel: Im November 2002 veranstaltete der (säkulare) Türkische Bund Berlin-Brandenburg (TBB) eine Gedenkveranstaltung zum 10. Jahrestag des Pogroms von Mölln - bekanntlich hatten Skinheads dort das Haus einer türkischen Familie in Brand gesetzt, wobei drei Menschen im Feuer umgekommen waren. Die strukturellen und inhaltlichen Parallelen zum Gedenken an die Novemberpogrome waren offenkundig 9 : Hier begann die Gedenkveranstaltung am Mahnmal für die Opfer von Krieg und Gewaltherrschaft in der Berliner Neuen Wache, wo ein Blumengebinde abgelegt wurde; das Mahnmal erinnert bekanntlich nicht nur an die gefallenen Soldaten und die Bombenopfer, sondern auch an die Ermordung der Juden.

Die Veranstaltung wurde sodann im Roten Rathaus fortgesetzt, mit Mitgliedern des Berliner Senats und nicht zufällig auch mit Repräsentanten der Berliner Juden und der Mit-Initiatorin des Mahnmals für die ermordeten Juden Europas, der Journalistin Leah Rosh. Die Assoziation von Mölln (und anderen Orten anti-türkischer Gewalt) mit der Judenverfolgung war also offenkundig.

Safter Cinar, Sprecher des TBB, machte das weiterhin deutlich in seiner Ansprache. Er verglich Mölln mit der nazistischen Judenverfolgung und erinnerte an die Geschichte deutsch-jüdischer Beziehungen; damit wollte er deutlich machen, dass die Deutsch-Türken Deutsche sind. So betonte seine Rede die türkische Integration in die deutsche Gesellschaft und bewegte sich dabei hin und her zwischen einem Betonen des Andersseins und der völligen Teilnahme in der deutschen Gesellschaft. Denn hier nun sprang Cinar über in das Thema der Teilhabe: Er verwies auf Helmut Kohls Erklärung von der »Gnade der späten Geburt« und bezog dies auf deutsche Türken: weder gäbe es eine Gnade der späten Geburt, noch eine Gnade des anderen Geburtsortes. Türken, so Cinar, wenn sie denn in Deutschland wohnen bleiben wollten, seien mitverantwortlich für die deutsche Geschichte und sie müssten die deutsche Erinnerung mit übernehmen:

Wir müssen als Bewohner dieses Landes den Anteil an Verantwortung für die Verbrechen der Vergangenheit, der auf uns zu kommt - ich weiß nicht, wie dieser Anteil zu definieren ist - wahrscheinlich braucht er gar nicht definiert zu werden - wir müssen diesen Anteil an Verantwortung übernehmen und wir sind dazu auch bereit, diese Verantwortung zu tragen. Ich möchte das, meine Damen und Herren, so formulieren: es gibt keine Gnade der späten Geburt....und es gibt keine Gnade des anderweitigen Geburtsortes! (Safter Cinar, 23. November 2002, Berliner Rathaus)

9) Zur Entwicklung des Gedenkens an die Novemberpogrome von 1945 bis 1995 siehe Bodemann (1996). 
Hier und andernorts beobachten wir also ein Hin und Her über den Grat zwischen Opferund Täterseite: hier die Identifikation mit den geläufigen »Schattenseiten« der deutschen Geschichte als ein dezidiertes Bekenntnis zum Deutschsein; andererseits, widersprüchlicherweise, der Hinweis auf türkisches Anderssein vermittels eines dem jüdischen analogen Narrativs. So beziehen sich deutsch-türkische organische Intellektuelle, türkische Repräsentanten und deren Organisationen auf die jüdische Minorität, sehen deren Situation als ähnlich strukturiert, und benutzen andererseits jedoch das jüdische Narrativ negativ, um sich, kontrastierend zu Juden als »normale« deutsche Bürger darzustellen.

\section{Religiöse Türken und Judentum}

Soweit zur säkularen türkischen Seite; wie steht es aber nun mit religiösen türkischen Muslimen? Es ließe sich argumentieren, dass der TBB als wichtiger Vertreter der säkularen Seite eine Allianz mit Juden sucht und das jüdische Narrativ in seiner Substanz übernimmt; der Cemaat andererseits, also eine der religiösen Organisationen, benutzt das Judentum als Form. Er bezieht sich, und kopiert dabei, die jüdische institutionelle Struktur in Deutschland, um ähnlich gelagerte Anerkennung für religiöse Rechte und Privilegien zu erhalten. Der $\mathrm{Ce}$ maat, wie andere türkische Organisationen außerhalb der Türkei, befindet sich zum ersten Mal in der Minderheit, in der Diaspora, und ist gezwungen, nach Modellen für diasporisches Leben zu suchen. Die anti-deutschen Ausfälle einiger Imams sind ja gerade Beweis dafür, dass sie sich mit ihrer diasporischen Situation befassen und nach Wegen (auch Irrwegen) zu einer geeigneten diasporischen Form suchen. Dementsprechend schrieb unlängst der stellvertretende Vorsitzende des Zentralrats der Muslime davon, dass Muslimen eine »Theologie der Integration « fehle. Die alten Schriften gäben selten Auskunft darüber, wie man sich in nicht-muslimischen Gesellschaften verhalten solle ${ }^{10}$.

In dieser neuartigen, diasporischen, Situation versuchen nun der Cemaat und andere religiöse Gruppierungen, jüdische institutionelle Strukturen als Modelle zu übernehmen, wobei die jüdische Gemeinschaft freilich idealisiert und deren innere Konflikte und inneren Gegensätze übersehen werden. So bewundert ein Vorstandsmitglied des Cemaat, Juden anderweitig nicht unbedingt freundlich gesinnt, deren imaginierten starken Zusammenhalt:

Ich wünsche mir von Allah, dass kein anderes Volk die Schwierigkeiten haben wird, die das jüdische Volk erfahren hat, aber ich wünsche mir von Allah, dass er alle mit einer Solidarität wie die der Juden untereinander versorgen würde. Da ist eine jüdische Gemeinde, die für alle Juden spricht. Mein Herz wünscht sich, dass alle türkischen Organisationen unter demselben Dach zusammenkämen und gleichen Abstand zu allen deutschen Parteien halten würden. (Interview mit A. Y., Türkische Gemeinde zu Berlin, 8. Mai 2003)

Obgleich nun diese Wünsche des Vorstandsmitglieds vom Cemaat sich nicht erfüllt haben, hat dieser schon einmal seine Organisationsstrukturen denen der jüdischen Gemeinde entsprechend modelliert. Das fängt mit dem Namen an: Mit Cemaat, Türkische Gemeinde zu Berlin wurde offensichtlich, einschließlich mit der antiquierenden Präposition »zu«, dem Namen der Jüdischen Gemeinde zu Berlin entsprochen. Auch der Verband der Muslime auf Bundesebene nennt sich, dem jüdischen Zentralrat entsprechend, »Zentralrat der Muslime in Deutschland $\ll$.

Zudem verlangen Repräsentanten des Cemaat offen den Juden entsprechende religiöse Rechte. Staatlichen Stellen in Deutschland sollen etwa die religiösen und nationalen Merkmale sunnitisch-türkischer Einwanderer anerkennen. Deshalb verlangen der Cemaat und andere religiöse Organisationen das Recht auf rituelles Schlachten, des Essens von halal, also religiös zubereitetem Fleisch, die Erlaubnis zum öffentlichem Gebetsruf und Beerdigungen

10) Mohammed Aman H. Hobohm, zitiert in Die Welt, 16. November 2004. 
nach islamischem Ritus auf getrennten islamischen Friedhöfen und insbesondere den Status als Körperschaften des öffentlichen Rechts, entsprechend dem der jüdischen Gemeinden ${ }^{11}$. Damit wiederum verbinden sich Forderungen nach Religionsunterricht an Schulen, in Berlin etwa seitens des Cemaat $^{12}$, und das Tragen des Kopftuchs in öffentlichen Einrichtungen, entsprechend der jüdischen Kippa.

Gerade die türkischen Muslime werden nicht müde, Parallelen zu den Juden zu betonen. M. Y. etwa, in Deutschland aufgewachsen, ein aufgeschlossener Jurist und Rechtsberater der Milli Görüs, findet es selbstverständlich, auch mit Juden gemeinsam zu verschiedenen Themen zu arbeiten. In einem Fall nahm er an einem Rechtsstreit zum Schlachten nach islamischem Ritual in Nordrhein-Westfalen teil:

Ich habe mit [den Richtern] stundenlang gesprochen. Ich habe ihnen alle rationalen Argumente vorgebracht. Aber sie haben sehr hart gegen uns argumentiert. Meine Argumente gelten für sie nicht. Da fing der Rabbiner neben mir an, zu sprechen. Er sagte, »ihr Deutsche habt kein Recht, zu sprechen. Ihr habt meine Verwandten ermordet.« Da waren die Deutschen still. Sie konnten nichts dazu sagen. Er hat sie so runter gemacht, wenn ich in ihren Schuhen gewesen wäre, ich könnte diese Beleidigungen nicht ertragen und würde rausgehen. Aber das ist sein [des Rabbiners] Kapital ${ }^{13}$. Sie haben sechs Millionen ermordet, sie können nicht rausgehen während der Rabbiner spricht. Die Zeitungen würden einen Skandal draus machen. So haben sie dem Rabbiner bis zum Ende zugehört. Als wir das Gericht verließen, sagte ich ihm, »vielen Dank. So erreicht man etwas. Wir wissen nicht, wie man so etwas macht.« Er sagte zu mir, »ich weiß, dass sie einen Groll auf mich haben. Das sitzt in ihren Genen. Das beste, das ihr tun könnt, ist nahe bei uns Juden zu stehen. Zusammen können wir gegen die Deutschen angehen. Unser Wort gilt dort etwas, eures nicht.« (Interview mit M.Y., Rechtsexperte der Milli Görüs, 27. Juli 2004)

$\mathrm{Ob}$ es nun in dieser merkwürdigen Art und vermutlich stilisierten Darstellung geschieht oder nicht, auch andernorts werden Juden als Vorbilder gesehen und genommen. Dabei wird oft die eigene innere Fragmentierung und organisatorische Schwäche der vorgestellten jüdischen Geschlossenheit gegenübergestellt. Emine Demirbüken, deutsch-türkische Politikerin und Ausländerbeauftragte im Berliner Bezirk Tempelhof-Schöneberg beklagt sich über mangelnde politische Einigkeit unter türkischen Einwanderern, entwirft so unausgesprochen eine diasporische Position und bezieht sich dabei wiederum auf die jüdische Gemeinschaft. Sie betont, wie wichtig es sei, der deutschen Gesellschaft das wirtschaftliche und intellektuelle Potential der Türken darzustellen: »Die jüdische Gemeinschaft verbindet die wirtschaftliche Kraft mit ihrer intellektuellen Kraft. «Doch die Türken hätten hier auch wirtschaftliche Kraft und viele Leute, die zweisprachig seien. Warum zeigten sie ihre Stärke den Deutschen nicht, und warum drängen sie sie nicht, Türken ernst zu nehmen? Wenn das nicht getan werde, dann würden die Deutschen sie immer stereotypisieren als Leute, die nicht Deutsch lernen wollten, deren Frauen alle von Männern misshandelt und deren Töchter zuhause eingesperrt würden. Demirbüken meint, die jüngere türkische Generation habe heute mehr Bildung, bessere Sprachkenntnisse und gesellschaftliche Fähigkeiten als die vorige Generation; Deutsche würden die türkische Gemeinschaft dann ernst nehmen, wenn sie mit jungen Deutsch-Türken als ihre Gegenüber in den ethnischen Organisationen gegenüberstehen. So wie in den jüdischen Organisationen (angeblich) der Fall, sucht sie fähige junge Türken mit ökonomischem und sozialem Kapital, um in der türkischen Gemeinschaft ganz vorne zu stehen. (Emine Demirbüken, 4. März 2003)

11) Zur Gesamtaufstellung ihrer Forderungen, vgl. Islamische Charta des Zentralrats der Muslime in Deutschland (2002).

12) Einem Verband, dem dies bereits gewährt wird ist die Islamische Föderation Berlin (IFB), der es erlaubt ist, Islam-Unterricht in deutschen weiterführenden Schulen zu erteilen. Zur Zeit nehmen über 1600 Schüler, je zur Hälfte Jungen und Mädchen, Religionsunterricht. 74\% sind türkischer, 21\% arabischer Herkunft. (IFB 2004).

13) Im türkischen Original: »Ama bunun sermayesi o«. 


\section{Ein Trialog?}

Wir haben eingangs darauf hingewiesen, dass Soziologen selten danach gefragt haben, wie Einwanderergruppen und ethnische Minoritäten ihr Verhalten an anderen Minoritäten orientieren; dabei sind gerade diese interethnischen Bezüge eine Form, in der sich gesellschaftliche Strukturen reproduzieren; diese wiederum geben auch trotz aller Globalisierung den einzelnen Staaten, etwa in der Europäischen Union, ihren besonderen ethno-kulturellen Charakter. Wir haben gezeigt, dass sich die deutsch-türkische Führung an jüdischem Gemeinschaftsverhalten orientiert, und ähnliches geschieht vice versa auf der jüdischen Seite. Dabei darf die Teilnahme von Juden an türkischen Veranstaltungen wie der zu Mölln nicht überbewertet werden. Die jüdische Präsenz in türkischen Veranstaltungen ist weiterhin gering $^{14}$, und obwohl Juden ein wichtiger Bezugspunkt für Türken geworden sind, spielen Türken in jüdischen Debatten eine geringe Rolle - es sei denn gezwungenermaßen als Mitbetroffene von neo-nazistischen Attacken und als religiöse Gemeinschaft mit gelegentlich ähnlich gelagerten politischen und rechtlichen Forderungen. Dies bezieht sich vor allem auf die religiösen Bedürfnisse der staatlich sanktionierten Gemeinschaften.

Dennoch spielen heute einzelne Juden, selbst stark jüdisch engagiert, wichtige Rollen im Kampf gegen Rassismus, Neo-Nazismus und im Bestreben, zwischen Juden und Türken/ Muslimen engere Beziehungen zu knüpfen. Eine dieser Organisationen ist die Amadeu Antonio Stiftung, die nach brutalen rassistischen Übergriffen in Brandenburg von Annetta Kahane gegründet wurde; die andere ist der von Irene Runge gegründete Jüdische Kulturverein, der sich vor allem aus ostdeutschen Juden und russisch-jüdischen Einwanderern rekrutiert ${ }^{15}$. Vermutlich aufgrund ihres Geschlechts und ihrer ostdeutschen Biografie sind beide Frauen freilich im bezug auf die jüdische Führung in Deutschland peripher geblieben. Kulturell und vom Klassenhintergrund her bewohnen die meisten Türken und Juden noch verschiedene Welten in Deutschland. Die meisten - zumindest nicht-russischen - Juden finden sich in der Mittel- bis oberen Mittelklasse, zumeist mit höherer Schulbildung, wohingegen die Mehrzahl der Türken als Gastarbeiter nach Deutschland kamen.

Angesichts des Unbehagens in den Beziehungen zwischen Juden und Deutschen nach der Schoah stünden hierbei die Türken als die natürlichen Partner der Juden zur Verfügung, als die »anderen Anderen « in der deutschen Gesellschaft, freilich ohne die historische Bürde. Deutsche Türken sind auch nicht die Enkel der Täter und könnten so eine unbelastete Beziehung zwischen den beiden Minoritäten mitbegründen. Von jüdischer Seite hat Maxim Biller gelegentlich diese Solidarität zwischen den ethno-kulturellen Minoritäten in Deutschland beschworen (Biller 2001, S.86).

Auf dem literarischen Terrain ${ }^{16}$ haben sich deutsch-türkische Autoren ebenfalls längst auf Juden bezogen. In ihrem Roman Selam Berlin thematisiert Yade Kara (2003) eine unterschwellige Rivalität zwischen Türken und Juden, die sich für einige der Romanfiguren aus der angeblich attraktiveren Kultur der Juden ergibt, jüdisch-türkische Rivalitäten, die im Übrigen auch bei jüdischen Autoren wie Doron Rabinovici (1999) und Maxim Biller erscheinen. Maxim Biller wiederum wurde von Feridun Zaimoglu, dem Autor der (diasporischen) Kanak Sprak (Zaimoglu 1995) zum »herausragenden Dichter jüdischen Herzens « erklärt; es ist offenkundig, dass er sich dem »begabtesten Polemiker Deutschlands« nahe fühlt und sich etwas mit ihm identifiziert. (Zaimoglu 2002)

14) Mit Ausnahmen. Pogrome wie die von Mölln haben bei Juden auf der persönlichen Ebene großen Schock ausgelöst. In Bodemann (2005, S.22) berichtet ein junger Mann über diesen Schock.

15) Siehe hierzu Fußnote 5.

16) Siehe hierzu die wichtigen Arbeiten von Leslie Adelson (2000, 2005). 
Kein anderer türkischer Autor schließlich hat das türkisch-jüdische Verhältnis in Deutschland und die Vorbildfunktion der jüdischen Kultur und Gemeinschaft so scharfsinnig beschrieben wie Zafer Senocak, vor allem in seinem Roman Gefährliche Verwandtschaft (1998), dessen Erzähler eine jüdische Mutter und einen türkischen Vater hat. Der Erzähler bringt das positive Zusammenleben von Türken und Juden im Osmanischen Reich in Erinnerung, betont, dass Juden und Türken in Deutschland durch eine besondere Beziehung zueinander geprägt sind, und sieht im übrigen beide Gruppen in einem »Trialog « mit den Deutschen.

Auf welches zukünftige Szenario lässt sich nach alledem schließen? Zunächst ist offenkundig, dass die Entwicklung zur türkischen Diaspora in Deutschland in vollem Gange ist. Hinter dem Vorwurf, Türken bildeten »Parallelgesellschaften« in Deutschland, steht die noch dumpfe Vorahnung, dass sich mit diesen vorgestellten Parallelgesellschaften diasporisches Leben entwickelt. Türken sind heute eben nicht mehr stumme, auf Rückkehr fixierte Migranten, sondern entwickeln ihre Stimme als Bürger hier in Deutschland. Es muss Deutschen nicht immer angenehm sein, was in diesem tastenden Prozess, durchaus mit Irrwegen, gesagt, getan und geschrieben wird. Es liegt in der Natur der Sache, dass sie ihre eigene Identität auch mittels wenig kompatibler Werte zu bestimmen suchen.

Zweitens werden zunehmender Rassismus und Antisemitismus zu häufigeren gemeinsamen Initiativen jüdischer und türkischer Organisationen führen. Für die Türken ist das $\mathrm{Zu}$ sammengehen mit Juden attraktiv aufgrund der historischen Bedeutung der jüdischen Minorität in Deutschland und der geschichtlichen Erfahrung (und Vorstellung) in der Türkei. Auch Juden kann es nicht gleichgültig sein, ob sie als numerische Winzlinge mit einer millionenstarken Bevölkerung koalieren sollten oder nicht, vor allem in einem Klima, in dem die Schoah langsam in den Hintergrund rückt.

Schließlich sehen wir in Deutschland eine außerordentliche Konstellation. In den meisten klassischen Einwandererländern, aber auch anderen Ländern Europas, finden wir eine kleinere oder größere Zahl verschiedener Einwanderergruppen. Selten jedoch findet sich eine Einwanderergruppe, die den anderen zahlenmäßig haushoch überlegen ist. Und in keinem anderen Land spielt die jüdische Gemeinschaft eine quasi staatstragende symbolische Rolle. Diese beiden Gruppen werden nun in einen »Trialog « mit dem deutschen Umfeld gebracht und müssen dabei selbst ihre Beziehung zueinander definieren. Für die deutsche Gesellschaft ist dies ist eine völlig neue Situation, die sie in der Zukunft noch stärker prägen wird, und in einer historisch für Deutschland präzedenzlosen Form.

\section{Literatur}

Adelson, Leslie (2005): The Turkish Turn in Contemporary German Culture: Towards a New Critical Grammar of Migration, London, New York: Palgrave Macmillan.

Adelson, Leslie (2000): Touching Tales of Turks, Germans, and Jews: Cultural Alterity, Historical Narrative, and Literary Riddles for the 1990s', in: New German Critique 80, S. 93-124.

am Orde, Sabine (2002): Türken fordern Vorschule für alle, in: die Tageszeitung, 21. Februar, S. 7.

Beauftragte der Bundesregierung für Migration, Flüchtlinge und Integration (2000): Einbürgerung: Fair, Gerecht, Tolerant, http://www.einbuergerung.de.

Biller, Maxim (2001): Deutschbuch, München: DTV

Bir, Cevik/ Sherman, Martin (2002): Formula for Stability: Turkey Plus Israel, Middle east Quarterly, http://www.science.co.il/hi/Turkish/Articles/Bir-2002.pdf.

Bodemann, Y. Michal (1996): Gedächtnistheater: Die Jüdische Gemeinschaft und ihre deutsche Erfindung, Hamburg: Rotbuch Verlag. 
Bodemann, Y. Michal (2002): In den Wogen der Erinnerung. Jüdische Existenz in Deutschland, München: DTV

Bodemann, Y. Michal (2004): Unter Verdacht. Parallelgesellschaften und Anti-Islamismus, in: Süddeutsche Zeitung, 20. November, S. 13.

Bodemann, Y. Michal (2005): A Jewish Family in Germany Today. An Intimate Portrait, Durham: Duke University Press

Bodemann, Y. Michal (1996a): Jews, Germans, Memory, Ann Arbor: University of Michigan Press.

Bodemann, Y. Michal (2004): The German Jewish Communities and the New Russian Jewish Writers, Unpublished conference presentation on the Russian Jewish Diaspora after the Cold War, Brandeis University, 21-22 April.

Burgauer, Erica (1993): Zwischen Erinnerung und Verdrängung - Juden in Deutschland nach 1945, Reinbek bei Hamburg: Rowohlt Verlag.

Çınar, Safter (2003): Eine Bemerkung zu Herrn Brenner, Jüdisches Berlin 59, S. 4.

European Forum for Migration Studies (2000): Beck presents report on the Situation of Foreigners in Germany, in: Migration Report: Chronology Of Relevant News And Occurrences In The Area Of The Institute's Work, February. http://www.uni-bamberg.de/ ba6ef3/dfeb00_e.htm.

Evrensel Daily Newspaper European Edition (2002): Yüksek mahkemeden kurbana vize çıktı, 16. Januar.

Hanioglu, Sükrü (2001): Preparation for a Revolution: The Young Turks, 1902-1908, Oxford: Oxford University Press.

Hürriyet Tageszeitung, Europäische Ausgabe (2001): Brenner: Ortak noktalarimiz var, 17. Juli.

Hürriyet Tageszeitung, Europäische Ausgabe (2002): Hesabi Tutmadi, 10. Juni.

Jonker, Gerdien (2002): Muslime in Berlin, Berlin: Ausländerbeauftragte Berlin.

Kafesoglu, Ibrahim (1999): Türk-Islam Sentezi, Istanbul: Ötüken Nesriyat.

Kara, Yadé (2003): Selam Berlin, Zürich: Diogenes Verlag

Kermani, Navid (2004): Distanzierungszwang und Opferrolle, Die Zeit 48, S. 62.

www.kuratandin.net: Arap Milliyetçilisi Ve Türkler Hakkindaki Uydurmalar

Landesarbeitsamt, Statistisches Landesamt (1997): Arbeitslosigkeit bei Türken. Statistik. Eigene Berechnungen www.tbb-berlin.de.

Mendelsohn, Ezra (1993): On Modern Jewish Politics, New York: Oxford U. Press.

Migrantische Initiative gegen Antisemitismus (2003): Antisemitizmle Her Yerde Mücadele, Jüdisches Berlin, 6(59) S. 4.

Milli Gazete, internet edition, Israil Kudurdu 25. September 2005, www.otuken.org

Milli Gazete, internet edition, Israil kudurdu 25. September 2005, www.nihalatsiz.org

Rabinovici, Doron. (1997): Suche nach M., Frankfurt: Suhrkamp Verlag.

Rubin, B. (2001): Turkey is Israel's Best Neighbour, Jerusalem Post, 11. Juli.

Senocak, Zafer (2004): Vernunft und Religion, die Tageszeitung, 22. November.

Senocak, Zafer (1998): Gefährliche Verwandtschaft, München: Babel Verlag.

Shaw, Stanford J. (1991): The Jews of the Ottoman Empire and the Turkish Republic, New York: New York University Press.

Der Spiegel (2004): Allahs rechtlose Töchter. Muslimische Frauen in Deutschland, 47, 15. November.

Statistisches Bundesamt und Türkischer Bund Berlin Brandenburg (2003): Einbürgerungen in Deutschland, http://www.tbb-berlin.de.

Statistisches Bundesamt (2000): Ausländische Wohnbevölkerung nach ausgewählten Staatsangehörigkeiten von 1997 - 2002, http://www.integrationsbeauftragte.de/download/datentab2.pdf.

Swidler, Ann (2002): Cultural Repertoires and Cultural Logics: Can They Be Reconciled?, in: Comparative and Historical Sociology, Winter 2002, S. 1-2.

Tulgan, S.B. (2004): Geliebtes Istanbul, Jüdisches Berlin, 7(60), S. 13. 
Türkischer Bund Berlin Brandenburg (1997): Arbeitslosigkeit bei Türken. Statistik. Eigene Berechnungen www.tbb-berlin.de.

Türkischer Bund Berlin Brandenburg (2003): Einbürgerungen in Deutschland, http://www.tbb-berlin.de. Turkish Daily News Electronic Edition (1999): Israelis concerned over PKK revenge attacks, 18. Februar. Yilmaz, M. (2004): Keine No Go Areas in Kreuzberg, Jüdisches Berlin, 7(60), S. 12.

Zaimoglu, Feridun (1995): Kanak Sprak. 24 Mißtöne vom Rande der Gesellschaft, Hamburg: Rotbuch Verlag.

Zaimoglu, Feridun (2002): Gerechtigkeit für Maxim Biller, in: Die Welt, 16. Februar 2002.

Zentralrat der Muslime in Deutschland (2002): Islamische Charta, http://zentralrat.de/3035.php.

Michal Y. Bodemann;

Gökce Yurdakul.

Joint Initiative for German and European Studies,

Department of Sociology, University of Toronto, 725 Spadina Ave., Toronto, Ont., M5S 2J4, Canada. bodemann@chass.utoronto.ca 